\title{
Computer Assisted System for Detecting Pulmonary Embolism in Lungs
}

\author{
M.Sucharitha, PHV Sesha Talpa Sai, M. L.R. Chaitanya Lahari, P. Haseena Bee
}

\begin{abstract}
A pulmonary embolism (PE) occurs when a blood artery in the lungs becomes suddenly blocked, generally owing to a blood clot. PE is a frequent life-threatening illness that should be diagnosed as soon as possible. A novel approach for automatically detecting $P E$ in contrast-enhanced $C T$ images is suggested in this research. To identify $P E$, computerized tomography (CT) is the main test to capture images. It is quick test, incursive with good quality images, enhanced contrast and multi-sliced images can be obtained. Candidate identification, feature calculation, and classification are all part of the system. The major aims of candidate detection are to include $P E$ with even entire occlusions and to eliminate erroneous diagnosis of tissue and parenchymal disorders. When calculating characteristics, the location and structure of the pulmonary vascular tree, as well as the severity, form, and size of an embolus, are all taken into consideration. The ability of the CAD tool to identify emboli in the sectional and sub sectional pulmonary Arterial Tree (PAT) was examined.
\end{abstract}

Keywords: Computer Assisted Detection, Mediastinum, Pulmonary Embolism, Computerized Tomography.

\section{INTRODUCTION}

$\mathrm{P}_{\mathrm{E}}$ is a potentially fatal illness which makes its detection difficult. One or more pulmonary arteries are partially or completely blocked [1]. In instances of pulmonary embolism, mortality, morbidity, and hospitalisation are all greater in Europe and the United States. PE is expected to occupy third place in the causes for deaths by 2030, according to illness mortality. [2,3]. Chance of occurring PE is more when thrombus or embolus, popularly called as blood clot, develops in the pulmonary arteries (PA). When a clot enters the PAT, it goes through the lungs' arteries, reducing pulmonary blood flow. As a result of the increased resistance to the bloodstream, this condition is associated with an increase in artery pressure, breathing issues, chest ache, and other breath related diseases. It may also produce an infarct or a drop in cardiac function and result in hemodynamic

Manuscript received on October 04, 2021.

Revised Manuscript received on October 18, 2021.

Manuscript published on November 30, 2021.

* Correspondence Author

Dr. M. Sucharitha*, Department of Electronics and Communication Engineering, Malla Reddy College of Engineering and Technology, Hyderabad, India. Email: sucharitha_m2002@yahoo.co.in

Dr. P.H.V. Sesha Talpa Sai, Department of Mechanical Engineering and Director R \& D, Malla Reddy College of Engineering and Technology, Hyderabad, India. Email: polamrajusai@gmail.com

Ms. M. L. R. Chaitanya Lahari, Associate Professor, Department of Mechanical Engineering, Malla Reddy College of Engineering and Technology, Hyderabad, India

Ms. P. Haseena Bee, Associate Professor, Department of Mechanical Engineering, Malla Reddy College of Engineering and Technology, Hyderabad, India

(c) The Authors. Published by Blue Eyes Intelligence Engineering and Sciences Publication (BEIESP). This is an open access article under the CC BY-NC-ND license (http://creativecommons.org/licenses/by-nc-nd/4.0/) problems, heart malfunction followed by death. [4]. Immobility, heart problems, overweight, increased blood pressure, surgery or trauma, cancer, long standing pulmonary illness, hormone disorders, pregnancy, and illness history in the family are all factors that might lead to PE [5]. If a thrombus develops in one of the veins (deep vein thrombosis), it has the potential to break off and enter the circulatory system, embolize via the heart, and lodge in one of the pulmonary artery's branches. PE is difficult to diagnose since the symptoms are non-specific and may not be present in all individuals. Although biomarkers and laboratory tests like ECG and determining the amount of cross-linked fibrin in the blood are useful in predicting the risk of PE, the diagnosis has basis on radiographic data from medical image processing techniques [6]. Each approach has its own set of advantages and disadvantages, and a move toward CT has been accepted. The first image capture method for determining the existence of $\mathrm{PE}$ was computerized tomography pulmonary angiography (CTPA) [7]. CTPA clinical studies revealed that 100 percent sensitivity and specificity were not reached [8]. As a result, misdiagnosis occurs, posing a health risk to patients and perhaps jeopardizing their lives. This study presents clinical data concerning PE as a disease, the method of computer-aided determination, and alternative segmentation strategies in detecting PE, as well as experimental findings of the novel method.

\section{LITERATURE}

PE may be lethal if left untreated, with a significant fatality rate that can be reduced by early identification [9]. Treatment comes in a variety of shapes and sizes [10]. The severity and prognosis of the PE dictate treatment choices. When just some sub sectional vessels are stopped, the obstruction is moderate; and several sectional or lobar arteries were occluded, the obstruction is severe. The responsiveness and particularity of CTPA scans ranges from 83 to 100 percent and 89 to 96 percent, respectively, according to a recent study of multiple CTPA clinical investigations. The working of 3 distinct CTPA-CAD setups from Philips, GE, and Siemens was investigated by Wittenberg et al. [11] and presented their findings "per clot." In Pulmonary angiography contrast agent is directly introduced to target arteries for a series of chest $\mathrm{X}$-ray projections, is a reliable procedure for detecting PE. "Single photon emission computerized tomography" (SPECT) [12] is a nuclear medicine technology that visualizes the distribution of radioactive material and provides an indirect method of embolus determination. To lower the FP rate, Al-Hinnawi et al. [13] proposed a 3D approach that is easier compared to neural networks and integrates preparation and evolution on dataset properties.

Published By:

Blue Eyes Intelligence Engineering and Sciences Publication (BEIESP)

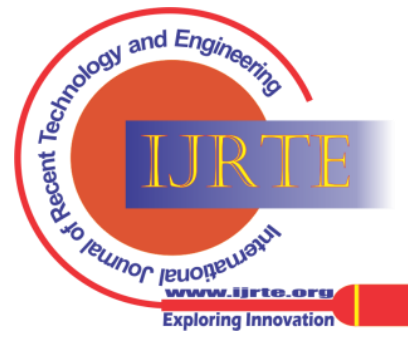




\section{Computer Assisted System for Detecting Pulmonary Embolism in Lungs}

Vascular Doppler ultrasonography [14] is a rapid, noninvasive, and accurate procedure. It is both painless and risk-free. However, compared to other imaging modalities, it delivers less clinical information.

To identify pulmonary nodules in CT scans, a novel technique [15] based on deep convolutional neural networks has been developed. By screening targets before detection, this strategy may be seen as an alternative method of lowering the false-positive rate of determination. A pulmonary embolus is one of the most life-threatening causes of chest pain, and it should always be evaluated when a patient complains of pain in chest and breathing problem to a healthcare practitioner. As a result, a CAD system is presented that can monitor Pulmonary Embolism using computer tomography pictures automatically.

\section{PROPOSED METHOD}

The CAD system is divided into numerous phases, each of which is discussed below. The first phase involves segmenting pulmonary arteries and detecting PE candidates within the segmentation. The major aims of the candidate-detection stage are to include PE and to eliminate erroneous determination of lymphoid tissue and parenchymal disorders. Following that, each candidate's characteristics are computed in order to categorize them. The intensity, form, and size of an embolus are all taken into account in feature calculation, as well as pulmonary vascular tree geometry and its placement within it.

Classification is used in the last step to differentiate between candidates who represent actual emboli and those who do not. The feature and classifier choices in the system have been enhanced. The vessel-segmentation strategy aims to restrict the candidate detection zone, which includes emboli, while avoiding false positives and lookalikes. PE has a number of look-alikes, including lymphocytic tissue, parenchymal disorders, and partial-volume voxels on the vascular boarder. Important feature of the technique is the 'sudden stop' (totally embolized arteries), which are often overlooked by existing sectional methods. On CT scans, the two biggest dark patches on thorax with a marginal 30 percent overlay extended over the transverse axis may be used to choose a preliminary lung region. These areas relate to the parenchyma of the lungs.

A morphological closure of $2.4 \mathrm{~mm}$ was utilized to eliminate gaps from the segmentation and include tiny vessels, which looked to include most small vessels. The mediastinum was segmented using a row-by-row method in which each pixel was placed between the lungs to determine the biggest area between them. To achieve smooth mediastinal segmentation $6 / 0 \mathrm{~mm}$ spacing is used. To keep tissue out of lung sectionalising, $11 \mathrm{~mm}$ geodesic distance with mask intensity greater than -300 $\mathrm{HU}$ is maintained and voxels at the mediastinum are eliminated. The principal pulmonary arteries are distinguished from other items in the lungs by expand segmentation to the mediastinum, region growth is used. Due to varying contrast enhancement, the limit is adjusted for all values in the data. It is chosen as the zero HU average and the more general force over the area for intensities over $+150 \mathrm{HU}$. employing a threshold because they seem brighter. To

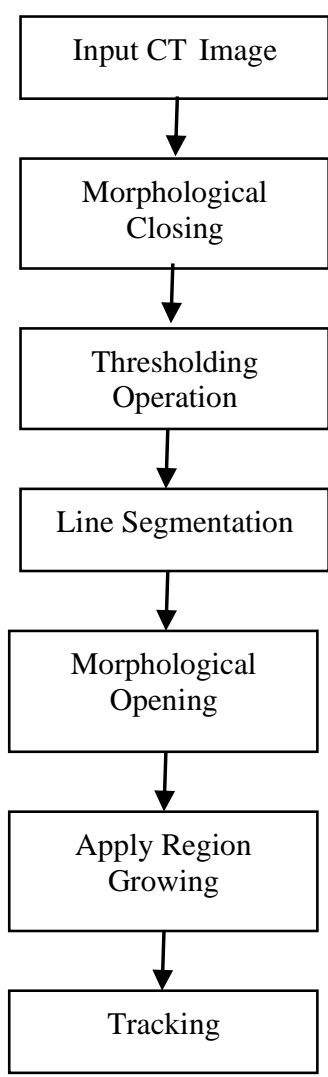

Fig.1. Proposed Pulmonary Embolism Detection

For small pulmonary vessels, the high critical value employed for large vessels is insufficient. The minimum intensity is related to the point-spread function (PSF), that creates difficulty in distinguishing vessels from (lymphoid) tissue based on intensity. The majority of the tissue lies towards the mediastinum, while small arteries are positioned in the circumference of the lungs. Peripheral-vessel sectionization is defined as a zone within the lungs with more intensity over $-150 \mathrm{HU}$. Lung parenchyma with a radius of less than $2.4 \mathrm{~mm}$ is removed to keep out big vessels, and is at least $15 \mathrm{~mm}$ away from the mediastinum to keep out tissue near the mediastinum.

The goal of tracing is to close any occlusion gaps that may exist between circumferential arteries and the remainder of PVT. The sectorizations of circumferential and main vessels are linked in healthy vessels without emboli. However, in individuals with PE, the blockage might be so substantial that a peripheral vessel's segmentation is disconnected from neighboring vessels. Because they have the same intensity, it may be tough to tell the difference between an embolized vessel and tissue locally. As a result, we're employing a tracking algorithm to figure out the best way to link the disconnected and other boats. Three input segmentations are used by the tracking method. The main tree contains all main and circumferential arteries linked with the mediastinum, is segmented first. The detached vessels are the second segment, and they include all main and circumferential vessels aren't linked to the mediastinum.

Published By:

Blue Eyes Intelligence Engineering and Sciences Publication (BEIESP)

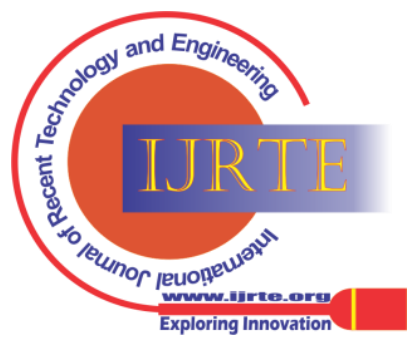


The third one is a look out or search region with a $-150 \mathrm{HU}$ intensity that is discovered within and between the lungs. Through the third region, the tracker connects the unlinked vessels of second region to the main tree search area of first region.

Based on intensity and distance, movement of wave-front is utilised to calculate expenses for every voxel. The cheapest paths between disconnected vessels and the leading tree are chosen to connect them. The candidate identification search area is formed by combining the dilated region around this route with the sectorization of the leading and circumferential vessels using an OR technique.

Candidate identification and feature calculation additionally employ segmentations of the tracked route and disconnected vasculature. Candidate identification is the process of extracting a group of voxels from the PA that include an embolus. The search area is lowered by identifying voxels and arranging related voxels into candidate items. It allows for more effective feature computing, while the organizing enables for object categorization.

Inside the vascular segmentation, candidate voxels are recognized in four different methods. The addition of two binary masks obtained by tracking: the mask of disconnected vessels and the mask of the dilated route connecting these vessels and the leading tree is one of them. Using an OR-operation, both masks are mixed with the other masks with new diagnosis methods. The other three techniques are based on the intensity level. The first one incorporates CT value within the vessels of CTA pictures directly. Hessian matrix eigenvalues are incorporated in the second step using second-order derivative matrices. If the eigenvalues are ordered by decreasing magnitude $\left(\left|\lambda_{1}\right|>\left|\lambda_{2}\right|>\left|\lambda_{3}\right|\right]$ a $+v e$ initial eigenvalue of the Hessian may be used to identify a dark patch.

$$
c_{\mathrm{z}_{2}}=\left\{\begin{array}{c}
\lambda 1, \lambda 1>0 \\
\lambda 3, \lambda 1<0 \text { and } \lambda 33>0 \\
0, \text { Otherwise }
\end{array}\right.
$$

With voxels at $\sigma=0.9 \mathrm{~mm}$ in each direction, Gaussian derivatives are utilised since they are the smallest scale that allows us to take accurate derivatives on our data.

The third choice is the grey scale, which features $4 \mathrm{~mm}$ dilations and $2 \mathrm{~mm}$ erosions to help with the transition from bright-enhanced blood to dark PE. The scope of the search is limited and PE candidates are found prior to this stage. The frequency of false positives, on the other hand, is still too high to be used as a CAD system output.

Erroneous detections are most often caused by parenchymal diseases, partial-volume impact on the vascular border, gaps in veins, noise, and motion artefacts. Candidate objects produce tools to allow for candidate classification to distinguish between true PE and look-alikes. The following statistics were obtained for each candidate's linked component: The mean, standard deviation, minimum 5th percentile and maximum 95th percentile are all numbers that may be used to calculate anything. The coverage \% is used to compute the relative size (size) of characteristics that are not measured on the whole object. Other criteria have been uncovered, and these are discussed further down. sub-optimal contrast enhancement, lymphoid tissue, flow

\subsection{Features}

\section{A. Intensity}

For categorization, three intensity-based metrics were utilized for determination. PE images are all be distinguished using a CT scan. The eigenvalues react to the dark areas on the map. Through transformation, noise, minor flow holes, and other spots with a shallow intensity valley are decreased.

\section{B. Shape}

The first shape-based characteristic is isophote curvature, which shows the local form of a surface through points of equal intensity and may be used to analyze the geometry of a dazzling lumen on its edge. PE and tissue are not distinguished only on the basis of intensity. Therefore, shape characteristic, at $\sigma=0.9 \mathrm{~mm}$, is suggested to differentiate the transitions 'lumen-tissue' (LT) and 'lumen-PE (LPE)'. The surface of an embolus resembles a cup or a valley. Because of the significantly bigger intensity transition between tissue and parenchyma, the shape determination of the transitions 'LPE' and 'LT' is readily polluted. As a result, we should focus just on the crucial intensities. This is achievable because to erf-clipping, which preserves as much edge information as possible. The concave lumen surface may be detected at embolic sites because to isophote curvature. It will, however, react to motion artefacts and sites where arteries and veins intersect.

The circularity of the lumen is the second aspect that indicates its form. Healthy pulmonary veins have a round sample, but an embolus within the channel makes it non-round. Two typical techniques to assess circularity are the area-to-perimeter relationship and the ratio of Hessian eigenvalues. The eigenvalues produced from the lumen segmentation distance transform are used to suggest circularity. The distance transform makes it possible to analyze the shape of massive vessels.

The third form-based feature assesses the vessel's shape rather than the lumen's shape. Tubularity is assessed in peripheral (segmental and smaller) vessels. Vesselness or stringiness is often used to assess tubularity. We advocate utilizing stringiness to evaluate tubularity near PE candidates because the orientation estimate near stenosis and occlusions is aided by ordering eigenvalues by value. Because many emboli are detected in peripheral arteries, stringiness is significant for PE categorization.

\section{Location}

The space to parenchyma was calculated using a two-phase space transform to the area with an force below -250 HU, is the first feature that employs a candidate's relative location to another structure. On CTA images, pulmonary arteries are brilliantly encircled by dark parenchyma. The partial-volume impact distorts the vessel's barrier, resulting in intermediate grey values with intensities comparable to emboli. The blur effect does not generate dark blotches within vessels. As a consequence, the distance-to-parenchyma may be used to tell the difference between emboli and false vessel boundary detections.

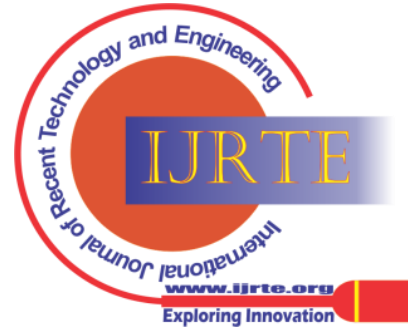


The distance to the mediastinum, which determines whether an embolus is detected in the mediastinum or in the periphery, is the second attribute which make use of candidate's location. As a consequence, new features may be used in the zone where they are optimised. The relationship of a vessel to large vessels, which is produced by the vessel-detecting program, is the third attribute based on a candidate's location. Vessel portions that are not linked to the mediastinum are recognised as suspicious areas or unconnected vessels. Because PE may generate an embolus, not only are disconnected arteries suspected of full embolization, but the monitored routes between an unconnected artery and the principle tree may also be required in identifying an embolus.

\section{D.Size}

A candidate is a category of interconnected voxels. Its size might be utilized to filter out erroneous positives. Noise and tiny flow holes may cause dark zones to appear everywhere. Areas with a moderate force valley may be avoided during classification; however certain noise-related valleys are extensive to be mistaken as embolus. A candidate is just a few voxels in size; the item's identification might be due to noise. As a result, tiny erroneous candidates are weeded out using size as a criterion.

\subsection{Segmentation ways}

\section{A. Body Segmentation}

The morphological operator closure is used to segment the body of the lung, and the holes are closed. In the discipline of mathematical morphology, closing is a crucial operator. It may be derived from the basic operations of erosion and dilation, much like its twin operator opening. It is usually used to binary pictures, like those operators; however grey level variants are available. Closing is similar to dilatation in that it enlarges the limits of foreground (bright) areas in a picture (while shrinking background color gaps), but it is less damaging to the original boundary form. A structural element, like other morphological operators, determines the particular operation. The operator has the effect of preserving background regions with a comparable form to this structuring element or that may entirely enclose the structuring element while removing all other background pixels areas.

\section{B. Lung Segmentation}

With the use of a thresholding process, the lung is segregated from the picture. The lung area is defined as a grey level value higher than 33. Individual pixels in an image are labelled as "object" pixels if their value is larger than a threshold value and as "background" pixels otherwise during the thresholding process.

\section{C.Mediastinum Segmentation}

The mediastinum was segmented using a row-by-row method in which each pixel was placed between the lungs to determine the biggest area between them. To achieve smooth mediastinal segmentation, a $0.6 \mathrm{~mm}$ morphological opening is used. To keep tissue out of lung sectionisation, all voxels near the mediastinum at $11 \mathrm{~mm}$ geodesic in a mask with intensity $>-300 \mathrm{HU}$ are eliminated.

\section{Vessel Segmentation}

The principal pulmonary arteries may be distinguished from other items in the lungs by employing a threshold because they seem brighter. To expand segmentation to the mediastinum, region growth is used. Due to varying contrast enhancement, this limit is adjusted for each data set. It is chosen as the $0 \mathrm{HU}$ average and the more common force in the area for forces over $+150 \mathrm{HU}$.

\section{RESULTS AND DISCUSSION}

The result of the CAD may be measured "per clot" or "per patient." The CAD results are recorded in proportion to the truth of all real PE events in the assessment on a "per clot" basis. The CAD does not need to search for all thrombi on a "per patient" basis. Figure 4 illustrates the PE for a Pulmonary artery image.
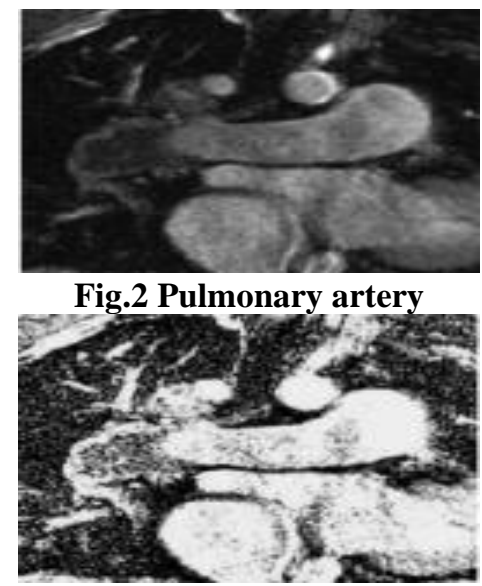

Fig.3 Segmented image

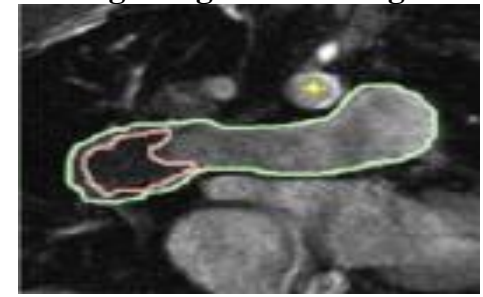

Fig.4 Pulmonary Embolism

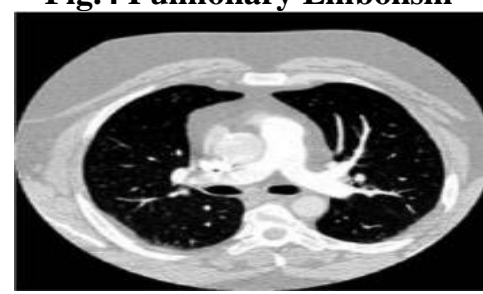

Fig.5 Input CT Image1

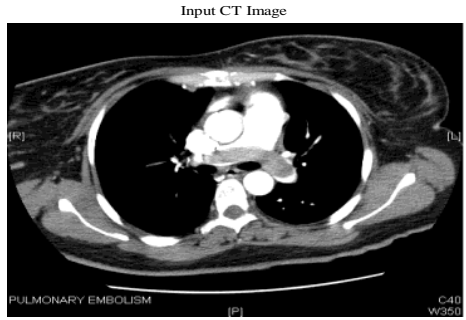

Fig.6 Input CT Image2

Published By:

Blue Eyes Intelligence Engineering and Sciences Publication (BEIESP)

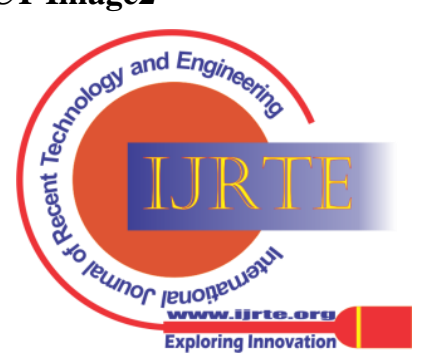


Figure 7 shows the segmentation produced for an example picture of figure 6.

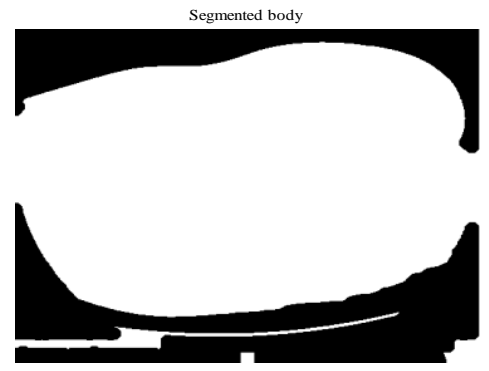

Fig.7 Segmented image

Only the Lung area is split in the CT scan, as illustrated in figure 8 . The divided mediastinum area depicts the zone containing the heart, which is distinct from other regions. The primary and peripheral vessels supplying the mediastinum are separated using a tracking algorithm, as illustrated in figure 10 .

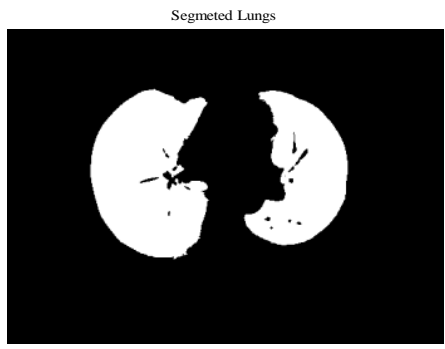

Fig.8 Segmented Lungs

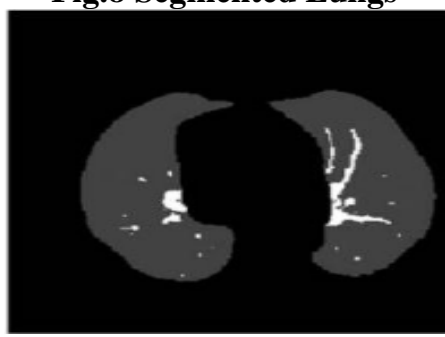

Fig.9 Segmented Pulmonary arteries

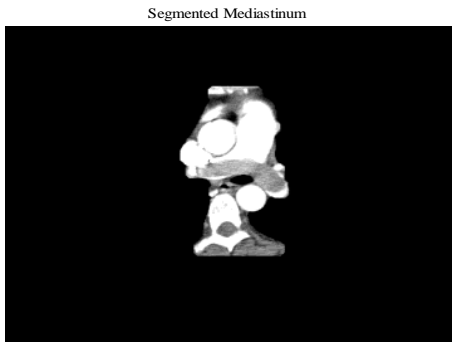

Fig.10 Segmented Mediastinum

Vessels are segmented after applying the region growing method and are depicted in figure 11.

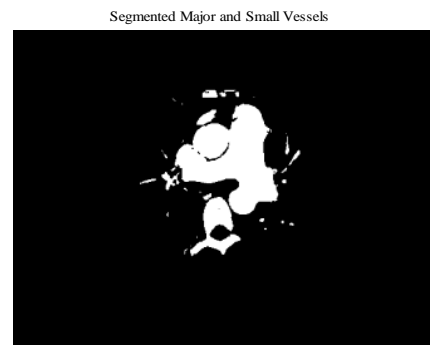

Fig.11 Segmented vessels

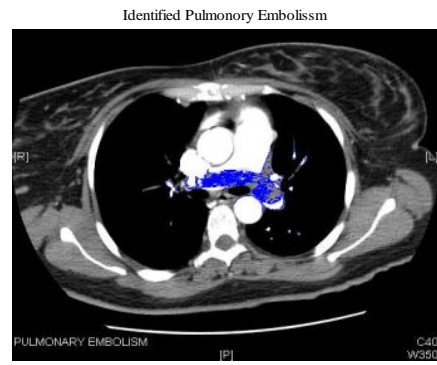

Fig.12 Pulmonary Embolism

The presence of Pulmonary Embolism is shown by the blue color zone in the above picture.

Table - I: Reference PE

\begin{tabular}{|c|c|c|c|}
\hline Data set & Occlusion & $\begin{array}{c}\text { Sub } \\
\text { segmental }\end{array}$ & Proximal \\
\hline \multirow{4}{*}{ UM } & $<20 \%$ & 33 & 52 \\
& $20 \%-80 \%$ & 94 & 170 \\
& $>80 \%$ & 118 & 128 \\
& All & 245 & 350 \\
\hline \multirow{3}{*}{ PIOPED } & $<20 \%$ & 33 & 53 \\
& $20 \%-80 \%$ & 153 & 279 \\
& $>80 \%$ & 96 & 186 \\
& All & 282 & 518 \\
\hline
\end{tabular}

Table I represents the standard PE identified by radiologists by artery level and arterial occlusion. The goal of early diagnosis of these areas is to lower the mortality rate of this illness that affects the lungs.

\section{CONCLUSION}

Pulmonary embolism is one of the reasons of sudden death and should be considered in people who have chest discomfort. Due to the existence of pulmonary embolus, the patient may collapse, cease breathing, and have their heart stop pumping, resulting in sudden death. The diagnosis of pulmonary embolism may be challenging, and it is often overlooked. From a computer tomography picture, our suggested method may automatically recognize the mediastinum area as well as the vessel region. The suggested computer-assisted detection methodologies enhance diagnostic accuracy and reduce the time it takes to interpret computed tomographic angiography for the identification of pulmonary emboli, allowing this test to be used as a first-line diagnostic modality in the case of suspected PE. The suggested Computer Aided Detection system has a high sensitivity for identifying peripheral pulmonary embolism, and it is unaffected by scanning circumstances or picture quality.

\section{REFERENCES}

1. Konstantinides. S.V, Torbicki. A, Agnelli.G, Zompatori. M, et al., "ESC guidelines on the diagnosis and management of acute pulmonary embolism", European Heart Journal,vol.35,pp.3033-3069, 2014.

2. Heit.J.A.,"The epidemiology of venous thromboembolism in the community", Arteriosclerosis, Thrombosis, and Vascular Biology, vol.28,pp.370-372, 2008.

\section{Published By:}

Blue Eyes Intelligence Engineering and Sciences Publication (BEIESP)

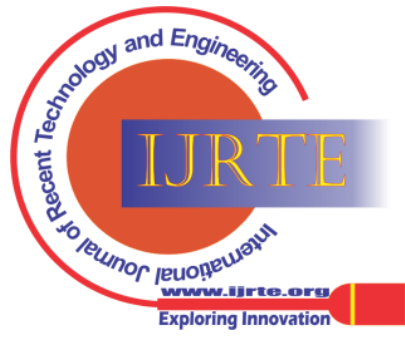




\section{Computer Assisted System for Detecting Pulmonary Embolism in Lungs}

3. Mathers. C.D and Loncar.D., "Projections of global mortality and burden of disease from 2002 to 2030", PLoS Medicine,vol.3,pp.11-17, 2006.

4. Wells.P.S, Ginsberg. G.S, Anderson. D.R, Kearon .C, Gent.M, Turpie.A.G, Bormanis.J, Weitz.J, Chamberlain. M, Bowie .D, Barnes .D and Hirsh. J., "Use of a clinical model for safe management of patients with suspected pulmonary embolism", Annals of Internal Medicine,vol.129, pp.997-1005,1998.

5. Goldhaber. S.Z and Morrison .R.B., "Pulmonary embolism and deep vein thrombosis", Circulation, American Heart Association,vol.12, pp.1436-1438, 2002.

6. Van Assen. H.C., "3D Active Shape Modelling for Cardiac MR and CT Image Segmentation”, Leiden University Medical Center, 2006.

7. Cronin.P, Weg.J.G and Kazerooni.E.A., "The role of multidetector computed tomography angiography for the diagnosis of pulmonary embolism", Seminars in Nuclear Medicine,vol38,pp.418-431,2008.

8. Dogan.H, de-Roos. A, Geleijins. J, Huisman. M.V and Kroft .J.M., "The role of computed tomography in the diagnosis of acute and chronic pulmonary embolism", Diagnostic and Interventional Radiology, vol.21, pp.307-316, 2015.

9. Armato. S.G, Altman.M.B and La Riviere P.J., "Automated detection of lung nodules in CT scans: Effect of image reconstruction algorithm", Medical Physics, vol.30,461-472, 2003.

10. Armato. S.G, Giger. M.L, Moran.C.J, Blackburn. J.T, Doi. K and MacMahon.H., "Computerized detection of pulmonary nodules on CT scans", Radiographics, vol.19,pp.1303-1311, 1999.

11. 11.Wittenberg.R, Peters. J.F, Weber. M, Cobben. L.P, Prokop.M, Schaefer-Prokop.C.M., "Stand-alone performance of a computer assisted detection prototype for detection of acute pulmonary: A multi-institutional comparison", The British Journal of Radiology,vol.85,pp.758-764, 2012.

12. 12.Hosch. W, Schlieter. M, Ley. S, Heye. T, Kauczor. H.U and Libicher. M., "Detection of acute pulmonary embolism: Feasibility of diagnostic accuracy of MRI using a stepwise protocol”, Emergency Radiology,vol.21,pp.151-158, 2014.

13. Al-hinnawi.A.R,Al-Naami.B.O and Al-Azzam.H., "Collaboration between interactive three-dimensional visualization and computer aided detection of pulmonary embolism on computed tomography pulmonary angiography views", Radiological Physics and Technology.vol.11,pp.61-72, 2018.

14. Park. S.C, Chapman.B.E and Zheng. B. A, "Multistage approach to improve performance of computer-aided detection of pulmonary embolisms depicted on CT images: Preliminary investigation", IEEE Transactions on Biomedical Engineering, vol.58,pp.1519-1527, 2011.

15. Jinglun Liang, Guoliang.Ye, Jianwen.Guo, Qifan Huang and Shaohui Zhang., "Reducing False-Positives in Lung Nodules Detection Using Balanced Datasets", Data-Enabled Intelligence for Medical Technology Innovation,vol.16,2021.

\section{AUTHORS PROFILE}

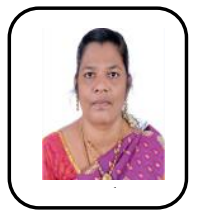

Dr. M. Sucharitha is working as a Professor of Electronics and communication Engineering Department in Malla Reddy College of Engineering and Technology, Hyderabad. She has completed her B.E. degree in Electronics and Communication Engineering in 2001 and M.E degree in Communication Systems in 2002. She received her Ph.D. degree in Information and Communication Engineering from Anna University, Chennai, in 2016. She has 16 years of academic experience, and apart from teaching, she was also involved in various activities for the betterment of student community as well as the institution. She started her research work in the area of image processing from 2009 onwards for adding more colors to her career. Her prime objective was to do research activity which should give benefits to the society. She was the project coordinator for three consecutive years in which she motivated and guided students to do project implementation in real field. Presently, four students are doing research under her guidance in the field of image processing. She is holding professional memberships in ISTE, IAENG, and IEEE. She is a proud recipient of Appreciation Award for her academic excellence in 2010 and also received 'Outstanding Woman in Engineering' award from Venus International and Young Researcher (women) award from SIAA-2019 for excellence in research activities.

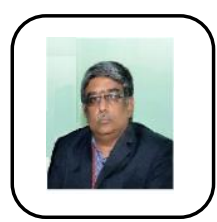

Dr. P H V Sesha Talpa Sai is presently working as Professor and Director, Malla Reddy College of Engineering and Technology (Autonomous). He obtained his Doctoral degree from JNTU, Hyderabad. He is having 16 years of industry and 16 years of academic and research experience. He is visiting faculty at Lincoln University College (LUC), Malaysia. He is guiding five Ph.D. Scholars from different Universities. He has 52 International and National Publications/Conference proceedings, 10 patents published, and 6 books/book chapters to his credit. His research interests are Nano Composite Materials for Solar Energy Conversion and Storage, Biofuels and Lubricants, Nanofluids in Energy and Heat Transfer applications, etc.

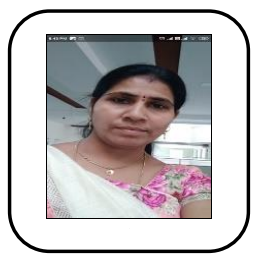

Ms. M L R Chaitanya Lahari is presently working as Associate Professor, Malla Reddy College of Engineering and Technology (Autonomous) and Research Scholar of School of Mechanical Engineering, REVA University, Bangalore. She obtained her B. Tech and M. Tech degrees from JNTU with distinction. She has 6 years of Teaching and Research experience apart from 2 years of industry exposure. She has 10 International and National Publications/Conference proceedings, three Patents, 3 books/book chapters to her credit, and guided many PG and UG students' projects. Her research interests are Nanofluids in Energy and Heat Transfer applications, Solar Energy Conversion and Storage Devises, Design and Management of Thermal Systems, Heat Transfer, Non-Conventional Energy Sources.

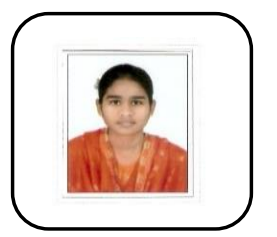

Ms. P Haseena Bee is presently working as Associate Professor, Malla Reddy College of Engineering and Technology (Autonomous) and Research Scholar of School of Mechanical Engineering, REVA University, Bangalore. She obtained her B. Tech and $\mathrm{M}$. Tech degrees from JNTU-Hyderabad with distinction. She has 05 International and National Publications/Conference proceedings, 2 Patents, 2 Books/Book Chapters to her credit and guided many PG and UG students' projects. Her research interests are Nanofluids in Manufacturing applications, Solar Energy Conversion and Storage Devises.

\section{Published By:}

Blue Eyes Intelligence Engineering and Sciences Publication (BEIESP) 94 (C) Copyright: All rights reserved.

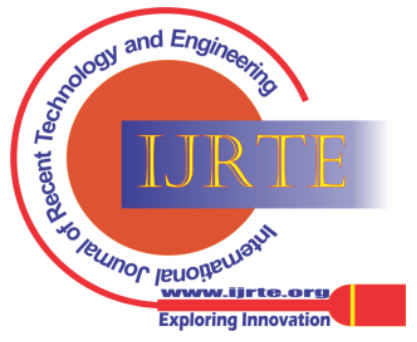

